\title{
A Review of the Psychological Support Mechanisms Available for Long-term Injured Footballers in the UK Throughout Their Rehabilitation
}

\begin{abstract}
Athletes regularly suffer psychologically as a consequence of long-term injury. However, to date there has been no investigation into the psychological protocols implemented to support long-term injured professional footballers. An online questionnaire was developed to identify the current rehabilitation practices in English football. 75 heads of medical departments responded to the survey from first teams and academies across the English Leagues. Medical staff recognised that there were clinical mental health problems, namely anxiety and depression affecting long-term injured players. It was acknowledged that there was a need for psychological support for long-term injured players. However, findings revealed most clubs had limited access to psychological practitioners and physiotherapy staff were almost entirely responsible for providing psychological support throughout rehabilitation. Clubs responded overwhelmingly that they wanted to be able to access psychological support for long-term injured players.
\end{abstract}

Keywords: Injury, psychological support, rehabilitation, football

\section{Introduction}

The risk of injury in English professional football has been reported to be 1,000 times greater in comparison to high risk jobs in industrial and commercial sectors (Hawkins \& Fuller, 1999). A study into injuries sustained in English professional football reported an average of 1.3 injuries per player per season (Hawkins, Hulse, Wilkinson, Hodson, \& Gibson, 2001). Major injuries (i.e. injuries that kept players from training or competing for four 
weeks or more) were found to account for $23 \%$ of all injuries sustained (Hawkins et al., 2001) indicating that long-term injury affects many players on a regular basis. There is a plethora of research demonstrating that athletes are psychologically affected by physical injury (Brewer, 2010; Nesti, 2010; Podlog \& Eklund, 2007; Putukian, 2016). Moreover, these difficulties are experienced immediately after sustaining a long-term injury, throughout rehabilitation and can persist beyond physical recovery (Podlog, Heil, \& Schulte, 2014). The question arises whether the psychological consequences of injury are routinely being acknowledged and supported.

Typically, there are three phases to the physical rehabilitation process for long-term injury (Prentice \& Arnheim, 2011). These are; Phase 1 - Acute, Phase 2 - Repair, Phase 3 Remodelling (or Return to training). In addition, with respect to sports performance, there is an additional phase (Phase 4) with regards to psychological rehabilitation referred to as Return to Competition (Clement, Arvinen-Barrow, \& Fetty, 2015). Importantly, these phases have different psychological impacts (Clement et al., 2015).

In Phase 1 athletes often exhibit debilitating emotional reactions such as shock, anger, fear, confusion, anxiety, and helplessness (Carson \& Polman, 2008; Tracey, 2003). The significance for a player experiencing prolonged negative emotional reactions should not be underestimated as this may result in increased risk of mental health problems. Phase 2 can be a long and drawn-out process of repairing physical damage (Heijne, Axelsson, Werner \& Biguet, 2008). Throughout this phase athletes can often experience psychological disruptions such as fluctuations in motivation, feelings of exclusion, and loss of athletic identity (Manuel et al., 2002; Ruddock-Hudson, O’Halloran, \& Murphy, 2012). Emotional reactions to rehabilitation include feelings of loss, frustration, denial, anger and depression (Clement et al., 2015; Tracey, 2003). Notably, research has demonstrated that these psychological responses to injury can trigger and/or uncover mental health issues such as depression, 
suicidal ideation, anxiety, eating disorders and substance use/abuse (Gervis, Pickford \& Hau, 2019; Heaney, 2006; Putukian, 2016; Smith \& Milliner, 1994; Sundgot-Borgen, 1994). The implication is that there is a need to provide psychological screening and support for athletes suffering from severe injuries to mitigate the risk of suicide. In phases 3 and 4 , fear of reinjury has been found to affect athletes' rehabilitation and post-injury performance. Fear of re-injury refers to anxious thoughts and feelings about an injury reoccurring when returning to sport (Tripp, Stanish, Ebel-Lam, Brewer, \& Birchard, 2007). This causes long-term physical impairment, reduced performance levels and satisfaction with sport and increased risk of further injury whilst training (Ardern, Taylor, Feller, \& Webster, 2012; Johnston \& Carroll, 1998; Lentz, Zeppieri, George, Tillman, Moser, Farmer \& Chmielewski, 2014).

Successful rehabilitation may be related to the degree to which medical staff meet the psychological needs of a long-term injured athlete (Heijne et al., 2008; Podlog \& Eklund, 2007). Despite research demonstrating that psychological interventions are effective in improving emotional states and the quality and rate of rehabilitation (Podlog et al., 2014; Santi \& Pietrantoni, 2013) the utilisation of psychological interventions in sport injury rehabilitation appears limited (Heaney, 2006). This may be related to the fact that injured athletes are generally only treated by physiotherapists, a group of professionals without psychology expertise (Arvinen-Barrow, Massey, \& Hemmings, 2014; Niven, 2007).

It has been argued that in order to care best for the needs of athletes throughout their rehabilitation, healthcare professionals should devote more attention to the psychological consequences of injury, in addition to the physical (Harris, Demb, \& Pastore, 2005; Podlog et al., 2014; Tjong, Murnaghan, Nyhof-Young, \& Ogilvie-Harris, 2014). Athletes felt that physiotherapists lacked awareness of the psychological challenges of injury and so were not in a position to provide motivational or emotional support (Arvinen-Barrow et al., 2014). 
In spite of the Football Association's aim to increase the awareness and application of sport psychology in English football (Pain \& Harwood, 2004) cultural barriers still appear to be blocking the implementation of psychological support mechanisms for long-term injured footballers (Heaney, 2006; Putukian, 2016). It has been suggested that, ideally, athletes should have access to a range of medical staff, including mental health professionals, throughout their rehabilitation (American College of Sports Medicine, 2006; Arvinen-Barrow et al., 2014; Brewer, 2010; Gervis, Pickford and Hau, 2018; NICE Guidelines, 2009).

Despite knowledge of the many negative psychological consequences of injury, physical treatment remains the norm for injured athletes (Tracey, 2003), with no, or inadequate focus on psychological consequences. Investigation into the provision of psychological support across different phases of rehabilitation is required.

The purpose of this research was to identify current rehabilitation practices in English football, to investigate if the psychological needs of long-term injured players were being considered and treated appropriately across all phases of recovery.

\section{Method}

\section{Participants}

In total, 75 heads of medical departments or equivalent positions with first teams $(n=42)$, academies $(n=33$, Cat. $1=21$, Cat. $2=10$, Cat. $3=2)$ from Premier League $(n=14)$, Championship ( $n=15)$, League One ( $n=3)$, League Two $(n=8)$, and Women’s Super League One $(n=2)$ football clubs in the UK participated in the study. Medics were specifically chosen because they could provide insight into the recovery process of long-term injured athletes and the specific services provided at each club to support those players.

\section{Measure}


An online questionnaire was designed to allow investigation and audit of the psychological support mechanisms currently available for long-term injured professional footballers. For the purpose of this study, long-term injury was classified as any injury that prevents a player from training for eight weeks or longer. The questionnaire consisted of 40 items and was organised into four sections: medical and support staff (13 items), screening for psychological issues (20 items), participants’ views on how psychological support should be provided for long-term injured players (3 items) and psychological support procedures (4 items). These items covered topics including: the number and type of support staff available for long-term injured footballers, which staff treated the players, psychological training of staff, psychological screening, psychological referrals, opinions about psychological support and whether or not clubs’ medical insurance covered mental health care. Questions were related to four different phases of rehabilitation from injury: acute (Phase 1), repair (Phase2), return to training (Phase 3) and return to competition (Phase 4). Although the questionnaire consisted of 40 items some items were skipped depending on the previous response. Different response formats were used; including categorical response questions (13 items), Likert scale (5 items, scored 1-5) (Simms et al., 2019), multiple response questions (14 items), and open ended questions (8), use of this variety of formats allowed for both quantitative and qualitative analysis.

\section{Procedure and Data Analysis}

Ethical approval was granted by the Research Ethics Committee. Contact details for the heads of medical departments were obtained from club websites. Email requests including a covering letter from the PFA explaining this is a joint research project were sent to relevant staff working at first teams and academies at each of the professional football clubs. Participants provided informed consent and completed the online questionnaire. Data were collected between June and September with reference to the previous football season. 
Data were analysed quantitatively and qualitatively as deemed appropriate.

Descriptive statistics such as percentages were tabulated for the categorical and multiple response questions. Chi-square tests were used where appropriate. For open-ended questions, data were first analysed by identifying all the responses and coding them into discrete categories. In many instances respondents identified more than one answer to the open-ended question, in such cases all responses were recorded. Subsequently frequencies in each category were calculated to determine the strength of each.

\section{Discussion of results}

The discussion of results combines both the quantitative and qualitative data. The data is presented under the following headings: Psychological issues associated with long-term injury, Who is treating long-term injured players? How are they treating them? Provision of psychological support. Results are discussed with reference to the four phases of rehabilitation.

\section{Psychological issues associated with long-term injury}

Perceived prevalence of psychological issues were identified by the question 'What are the most common psychological issues experienced by players suffering from long-term injury?'. Participants were able to give multiple responses to this open-ended question. The figure below illustrates the frequency of reported issues. 


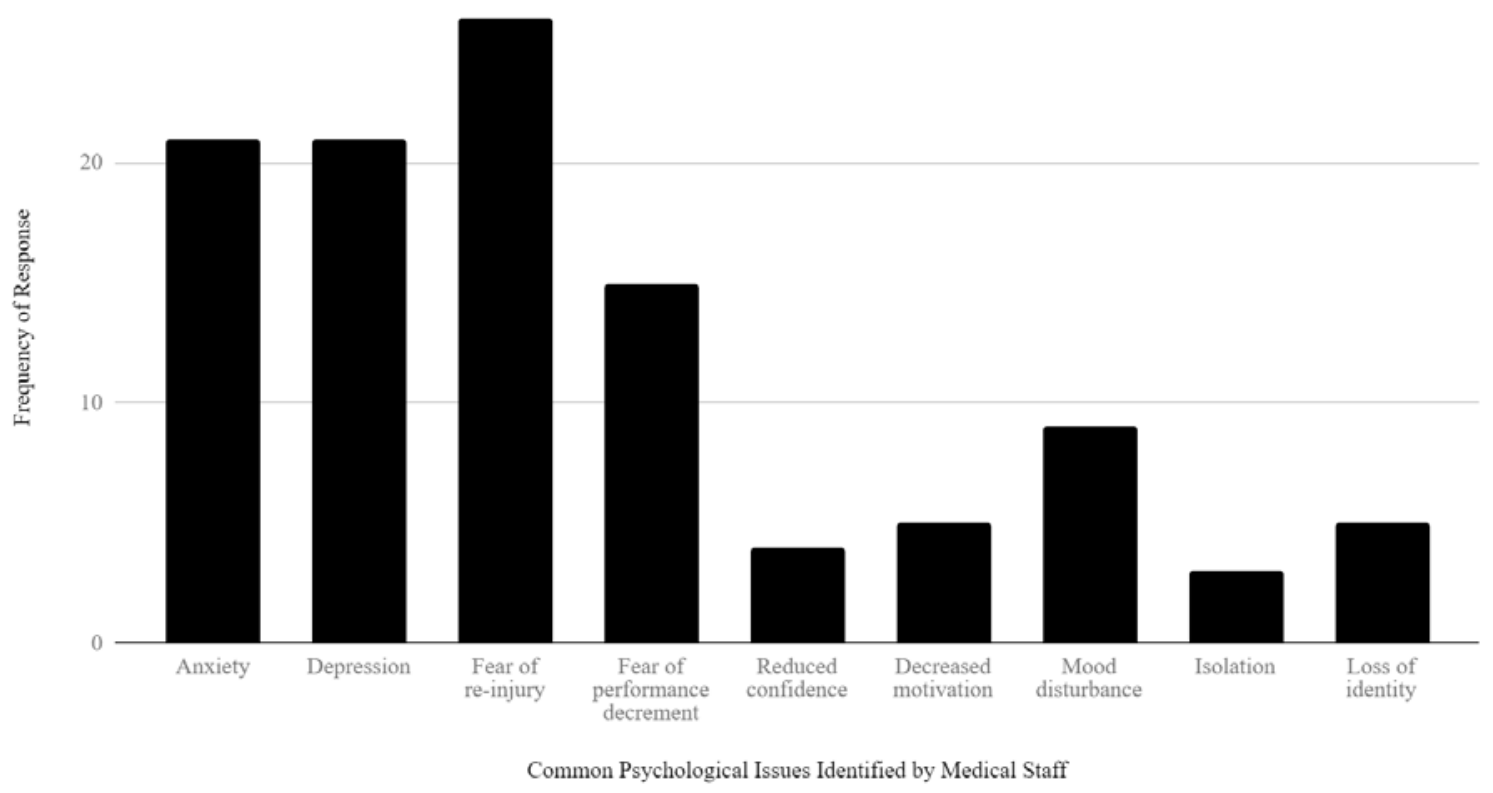

Figure 1. Most frequently identified psychological issues experienced by long-term injured players.

Figure 1 demonstrates that medical staff are aware of the psychological challenges faced by long-term injured players. Of the three issues most frequently identified, two can be considered clinical mental health problems, namely anxiety and depression. This finding aligns with the current body of research that has provided evidence of psychological vulnerability associated with long-term injury (Carson \& Polman, 2008; Clement et al., 2015; Tracey, 2003). Notably, this finding illustrates the awareness some medical staff have of these clinical issues, which is critical for ensuring players receive appropriate psychological treatment. Fear of re-injury was identified as the single most common psychological issue among long-term injured players, which concurs with previous literature (Ardern, et al., 2012; Lentz et al., 2014). However, substance misuse and addiction were not identified as common issues despite previous evidence to the contrary (Gervis, Pickford \& Hau, 2019; 
Heaney, 2006). Moreover, there were some clubs who did not identify any psychological issues associated with long-term injury as exemplified by one respondent who stated: ' $I$ never had any psychological issues that required professional input'. Given the plethora of evidence which demonstrates the prevalence of mental health problems associated with longterm injury, this statement speaks to the lack of awareness within support teams. The implication of this is that players are being put at risk.

Given the seriousness of the psychological issues identified, it was important to ascertain the perceived need for psychological support of long-term injured players.

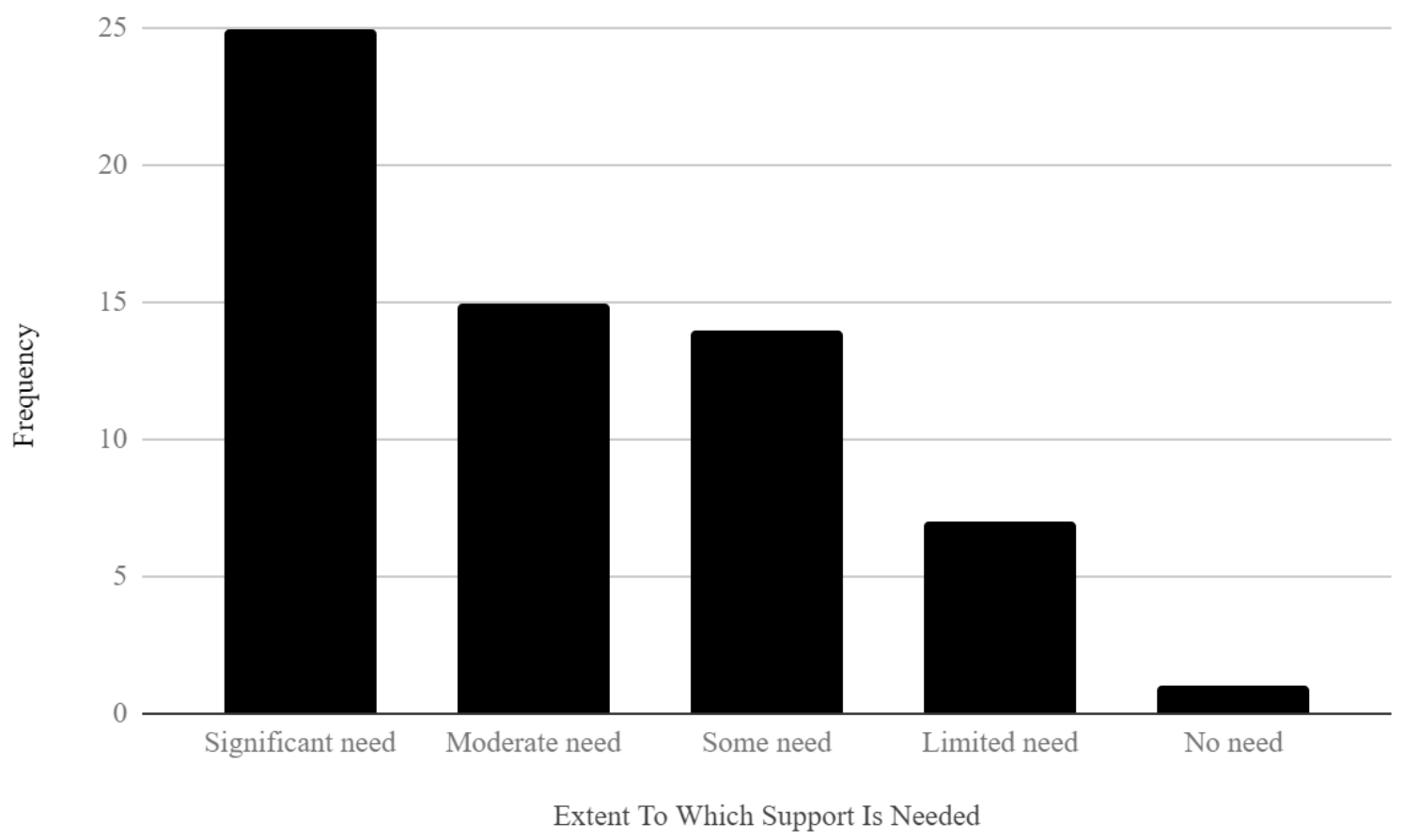

Figure 2. Perceived need for psychological support for long-term injured players

Findings reveal that the majority of clubs acknowledged a need for psychological support to a large extent, which aligns with recommendations (American College of Sports Medicine, 2006; Gervis, Pickford \& Hau, 2019; NICE, 2009). In order to understand 'who’ was available to treat long term injured players, clubs were asked to identify how many staff 
were employed in the categories below.

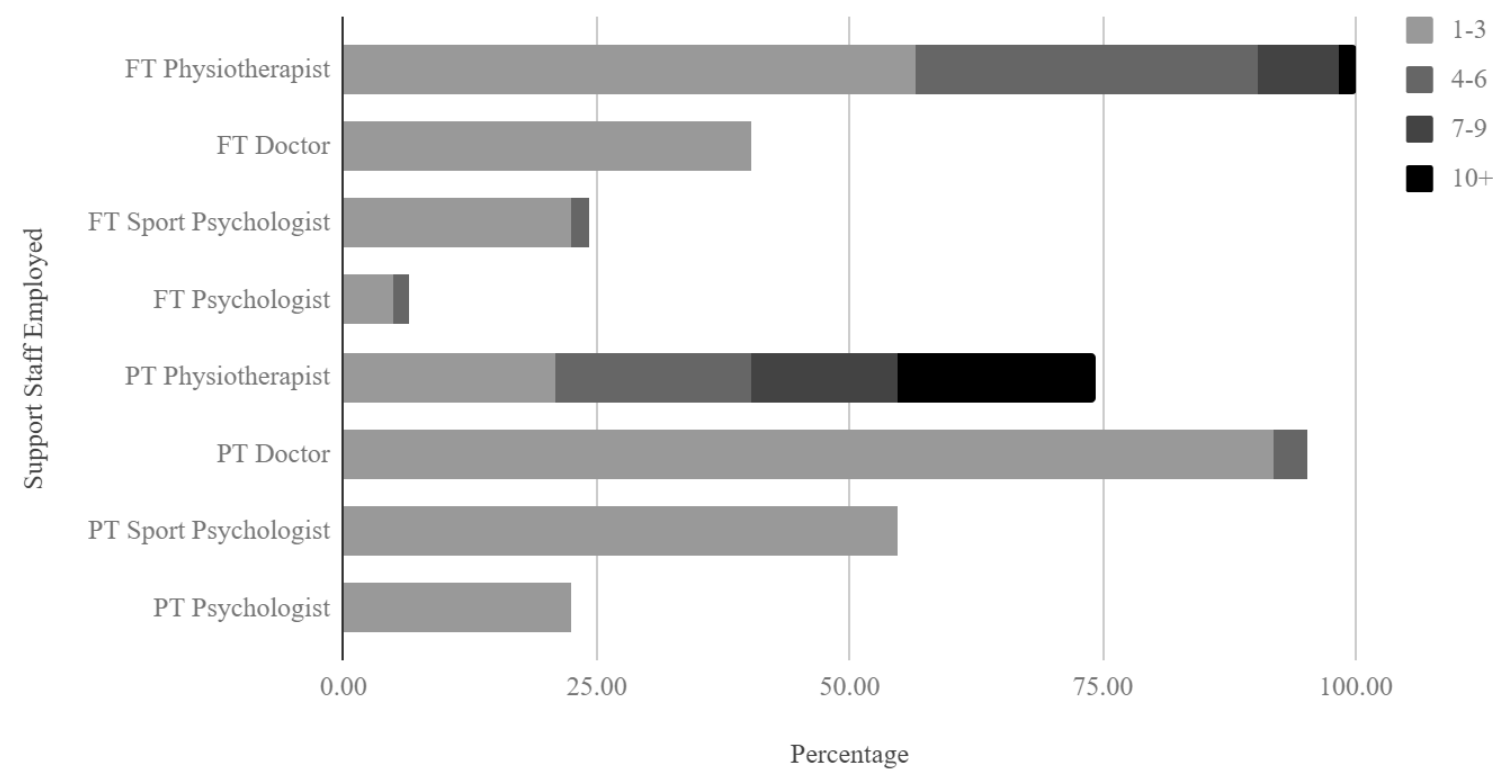

Figure 3. Support staff employed by clubs (\%)

The majority of staff who were available to treat long-term injured players were physiotherapists, with all clubs employing a significant number of full- and part-time staff. In comparison, relatively few clubs employed either full-time sport psychologists or counsellors. These results are in line with previous research which has found that treatment of long-term injury is almost entirely physical (Arvinen-Barrow et al., 2014; Tracey, 2003).

While there appears to be a satisfactory number of part-time sport psychologists employed by clubs, this finding may be explained by the Premier League’s Elite Player Performance Plan (EPPP, 2011). In order for clubs to receive funding academies must demonstrate the application of sport psychology. Indeed, 26 of 29 Academies (90\%) compared to only ten of 44 first teams (23\%) had access to a part-time Sport Psychologist. If part-time sport psychologists are only contracted to work at clubs once a week, once a month or once every few months they will not be able to provide regular treatment for all long-term injured players' rehabilitation. Moreover, it is unlikely that part-time sport psychologists will be able to provide regular, adequate and significant treatment for one or multiple long-term 
injured players if they are also working on other duties such as performance enhancement or organisational processes. However, a limitation of this question was that 'Part-Time' practice was not explicitly quantified, therefore it is possible that some of these individuals may only work a few hours each month.

\section{Who is treating long-term injured players?}

Table 1 shows the different professionals who treated long-term injured players across

Table 1

Type of Staff Treating Long-term Injured Footballers at Different Phases of Rehabilitation $(\%)$

\begin{tabular}{lcccc}
\hline & \multicolumn{4}{c}{$\begin{array}{c}\text { Phases of } \\
\text { Rehabilitation }\end{array}$} \\
\cline { 2 - 5 } Type of Staff & Phase 1 & Phase 2 & Phase 3 & Phase 4 \\
\hline Physiotherapist & 100 & 100 & 100 & 97 \\
Doctor & 64 & 60 & 47 & 47 \\
Strength \& & 39 & 85 & 85 & 80 \\
Conditioning Coach & 33 & 39 & 32 & 32 \\
Sport Psychologist & 33 &
\end{tabular}

In line with existing research (Niven, 2007; Tracey, 2003) physiotherapists were the central healthcare professional for long-term injured players. Almost every (98\%) first team and academy had access to full-time physiotherapists and almost every long-term injured player was treated by physiotherapists across all four stages of their recovery. This would seem to suggest that psychological treatment throughout rehabilitation is still being overlooked and supports previous research which suggests that physical treatment remains the predominate norm for injured athletes despite recommendations to the contrary (American College of Sports Medicine, 2006; Tracey, 2003). 
Furthermore, in response to the question 'Do you have staff trained in the psychology of injury?', only $37 \%$ of clubs reported that they had staff with relevant training, whereas the majority of clubs (55\%) did not have staff with this expertise. Therefore, it is unlikely that the medical staff treating long-term injured players would have the expertise to appropriately support players with the mental health concerns that were highlighted in Figure 1.

Indeed, where training was indicated, the vast majority reported only receiving limited formal education as part of a physiotherapy/sport science degree (22.6\% of total clubs). Whereas informal learning was cited as continued professional development (5.3\%), and 'on-the-job’ learning (10.6\%) thus supporting Arvinen-Barrow et al., (2014). These findings highlight the limited appropriate training of support staff in professional football clubs and raises questions about their ability to meet the psychological needs of their players. However, there would appear to be a lack of acknowledgement of these limitations and the potential risk to players as exemplified by this comment: 'Although we are not specifically trained in it, the medical/sport science team deal with the psychological issues with reasonable success; with the help of the coaches/club Chaplin as required'.

Furthermore, medical and even psychological staff treating injured athletes were not necessarily trained to identify, refer or treat many different mental health issues such as those identified in Figure 1.

\section{How are they treating?}

Initial psychological treatments always involve some specific method of screening to identify the potential psychological issues in order for treatment to be targeted and effective (Hsu et al., 2017). Where screening was reported, the majority was occurring in Phase 2 of rehabilitation (72.1\%). However, on average a third of clubs were not screening in any phase 
of rehabilitation. Figure 1 identifies the psychological screening practices of clubs across the four phases of rehabilitation.

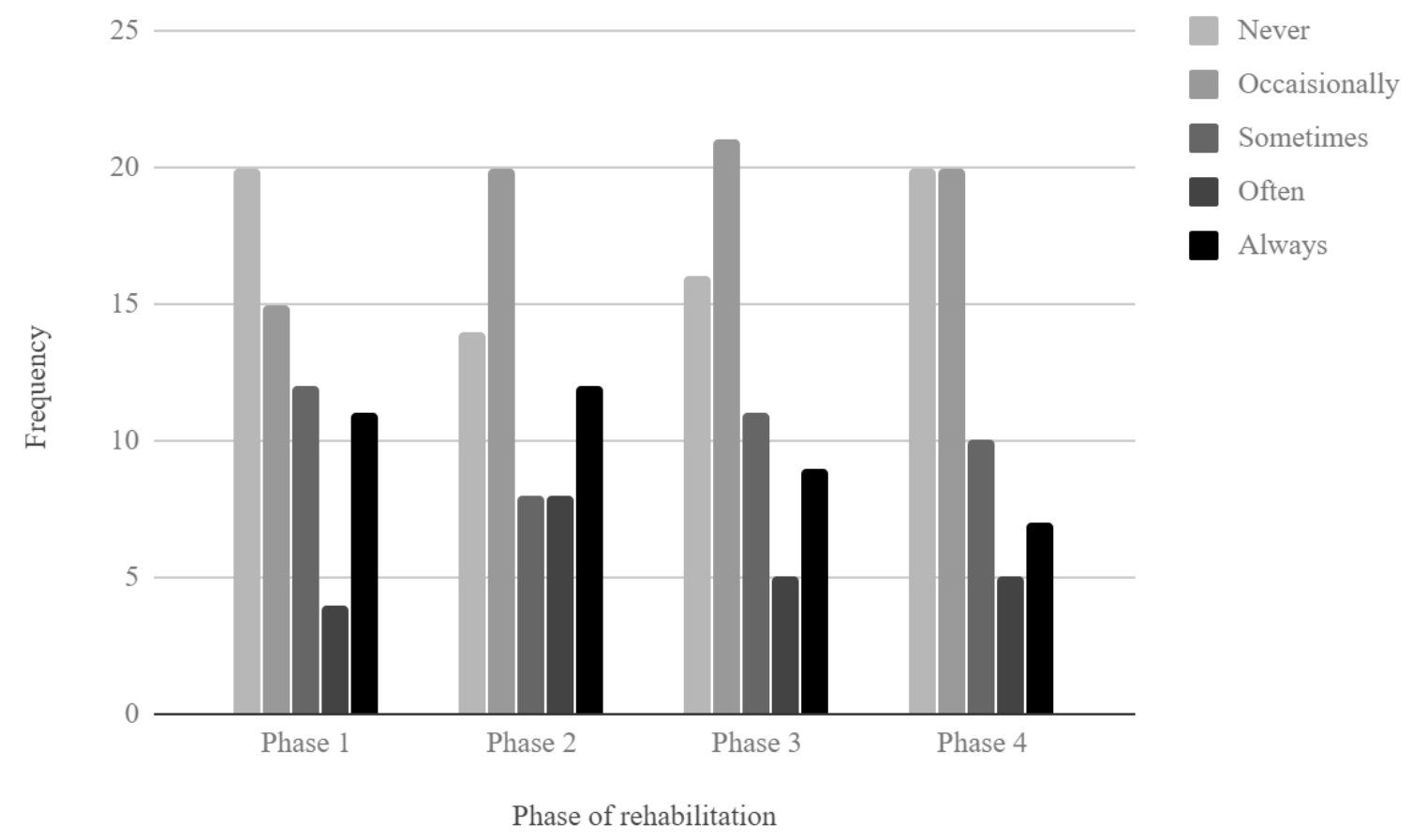

Figure 4. Amount of psychological screening across the four phases of rehabilitation (\%)

The majority of clubs (62.3\%) reported “never” or only “occasionally” screening for psychological issues throughout a player's recovery from injury leaving them vulnerable to experiencing problematic rehabilitations, problems with future performance, an increased risk of injury, not returning to competitive sport and triggering or uncovering mental health issues (American College of Sports Medicine, 2006; Ardern et al., 2012; Podlog et al., 2014; Putukian, 2016).

Figure 5 shows the different methods used by those clubs who reported any psychological screening, at any phase of rehabilitation 


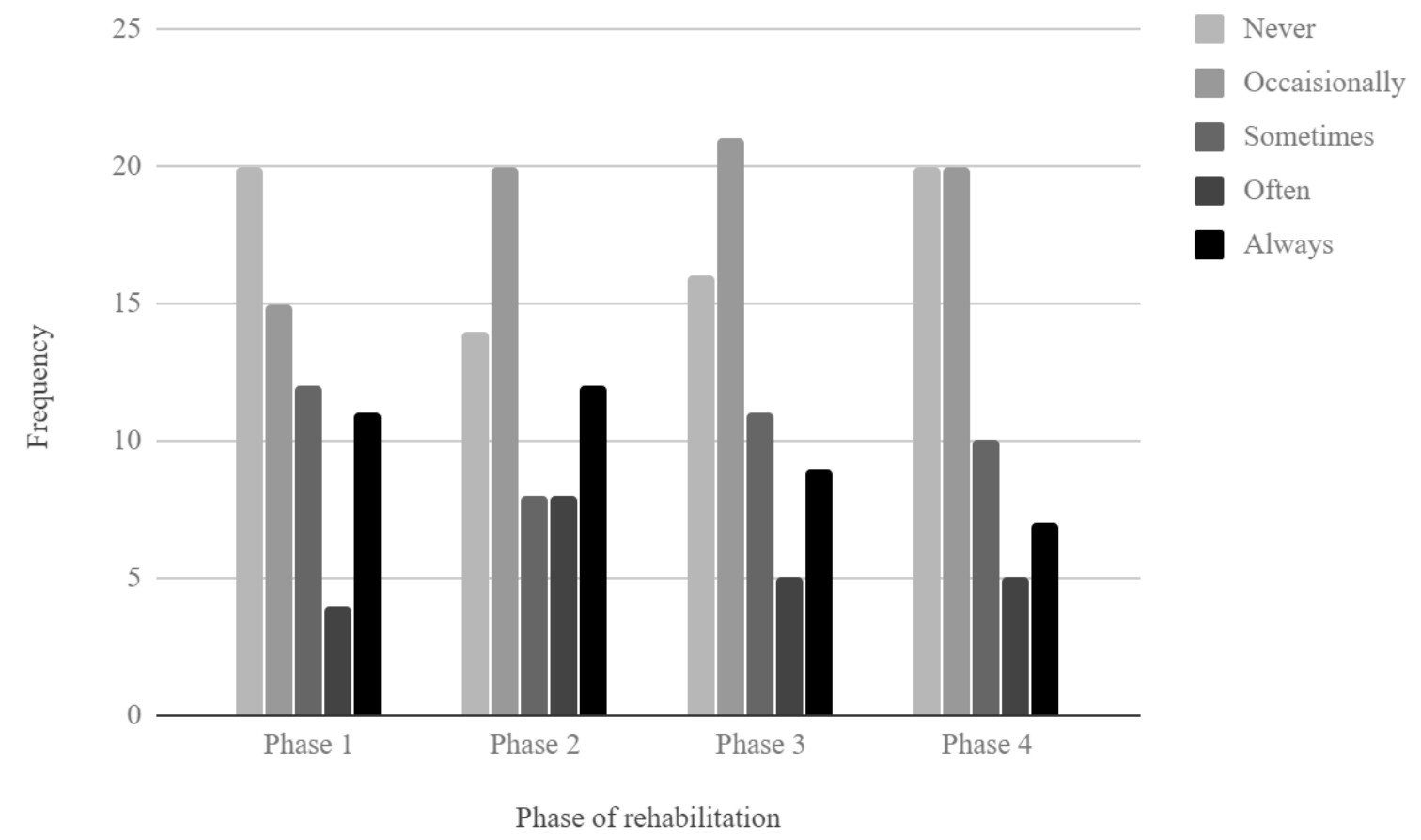

Figure 5. Psychological screening practices

The most frequently reported response was that screening was undertaken as part of the physiotherapist's assessment despite research showing that many physiotherapists do not believe they are adequately trained to identify or manage the psychological features of injury (Arvinen-Barrow et al., 2014).

All “other” methods referred to using individuals unlikely to be trained in the psychology of injury and/or using informal methods unlikely to be psychologically valid. For example, screening methods included: "Behavioural Strategist” and "Informal feedback from coaches”. Although the percentage of clubs screening for psychological issues appears low, the number of clubs effectively screening using psychologically validated screening methods may be even lower. Confusingly, of those who screened, the vast majority reported screening for clinical issues, see below.

Of the clubs who reported any psychological screening (68\%), Table 2 identifies what issues they reported screening for at the four stages of recovery. 
Table 2

Psychological Issues screened for at Different phases of rehabilitation (\%)

\begin{tabular}{lllll}
\hline & \multicolumn{4}{c}{ Phases of rehabilitation } \\
\cline { 2 - 5 } Psychological issues & Phase 1 & Phase 2 & Phase 3 & Phase 4 \\
\hline Fear of re-injury & 77 & 85 & 92 & 91 \\
Anxiety & 87 & 89 & 82 & 84 \\
Depression & 77 & 83 & 50 & 53 \\
Substance Misuse & 9 & 13 & 6 & 4 \\
Eating Disorder & 15 & 20 & 10 & 9 \\
Suicidal Ideation & 15 & 17 & 10 & 9 \\
Addiction & 6 & 6 & 2 & 2 \\
\hline
\end{tabular}

In the main, these screening practices reflected the most common issues cited by participants (see Figure 1). Table 2 indicates that the majority of those who screened for psychological issues, screened for fear of re-injury, anxiety and depression throughout a longterm injured player's recovery from injury. In comparison, the majority of clubs did not screen for other damaging mental health issues such as; substance misuse, eating disorders, suicidal ideation or addiction.

Given the responses outlined in Figure 5, it is questionable if these screening procedures are valid and reliable. For example, seventy-seven percent of clubs screened for fear of re-injury immediately after injury. Given that fear of re-injury occurs when athletes return to training or competition, in Phases 3 \& 4 (Podlog \& Eklund, 2006; Tripp et al., 2007), screening for fear of re-injury in Phases $1 \& 2$ is redundant. Hence, current screening practices seem to highlight medics' lack of understanding, and may reflect their lack of training. Therefore, further investigation of how these psychological issues are screened for is warranted.

Chi-squared tests were performed to examine the relationship between how clubs reported screening for psychological issues and which staff treated players across the four 
different phases of recovery. Significant relationships were found between how often psychological issues were screened for and who treated the player in Phase $1 \chi^{2}(48, N=75)$ $=83.07, \mathrm{p}<.01, V=0.61$, Phase $2 \chi 2(72, \mathrm{~N}=75)=110.73, \mathrm{p}<.01, V=0.70$ and Phase $4 \chi 2$ $(72, \mathrm{~N}=75)=106.03, \mathrm{p}<.01, V=0.69$. With regards to this relationship it was interesting to find that all clubs who "never” screened for psychological issues at Phase 2 had no psychological professional in their treatment staff. In comparison, 83\% of clubs who “always” screened for psychological issues in Phase 2 treated players with a combination of staff that included a sport psychologist. This same trend was observed in Phases 1 and 3.

The implication of this is that where clubs do not screen there is an increased risk for players with mental health needs to go untreated. This is further evidenced by the positive correlation found between screening practices and the number of referrals, $r s(73)=.43, p<$ .01 such that when clubs reported 'always' screening, they also referred more players to mental health specialists.

\section{Provision of psychological support}


Finally, if participants reported a need for psychological support they were asked how it should be provided, (Figure 6).

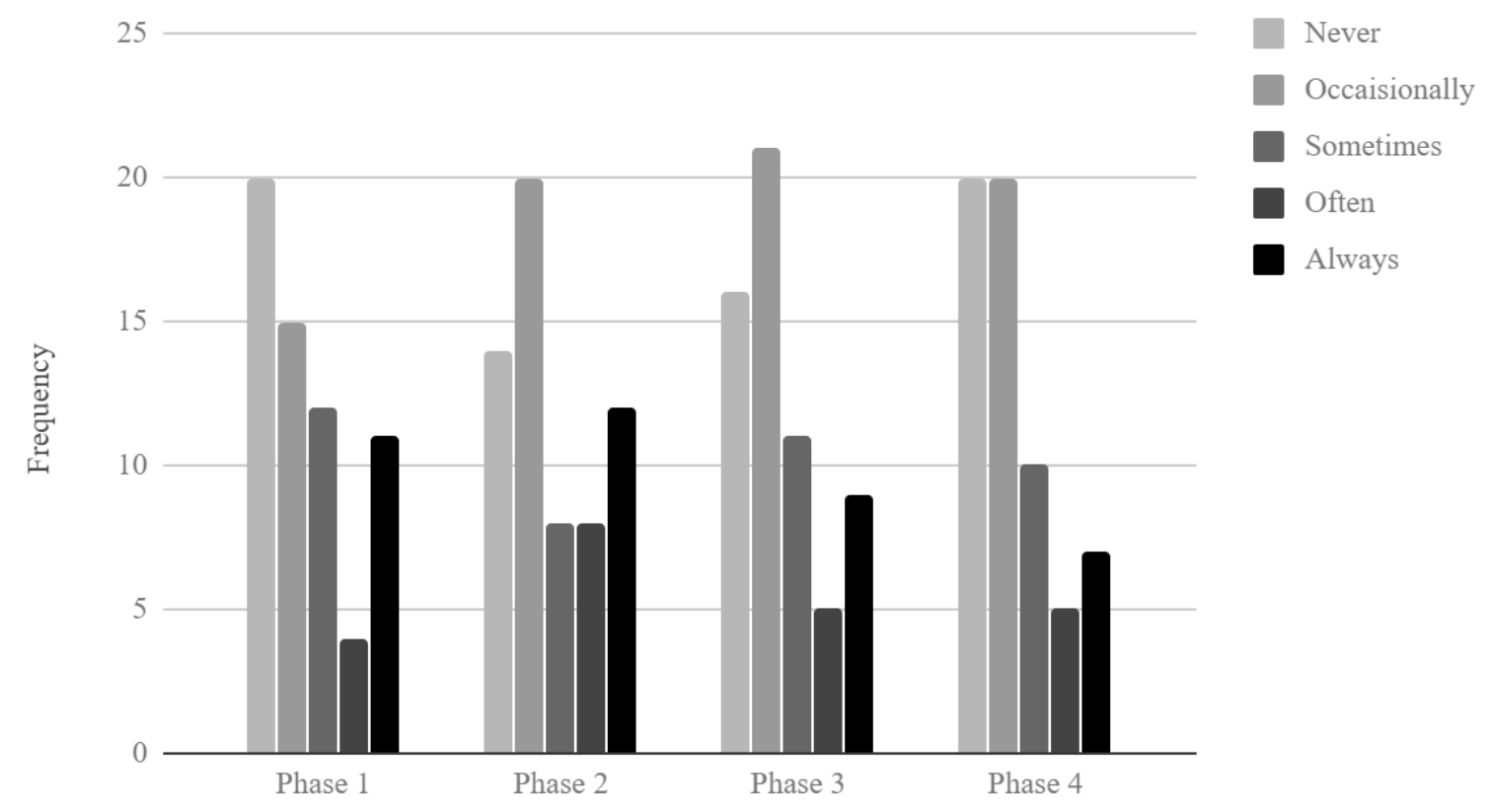

Phase of rehabilitation

Figure 6. ideal methods of psychological support for long-term injured players (Frequency)

From the 65 clubs that responded to this question, overwhelmingly they reported wanting to be able to access some kind of professional psychological support; either from a sport psychologist or through referral to other mental health specialists. As illustrated by one respondent; 'psychologists should be present at the club for the players to access with ease'. This aligns with the recommendations from the American College of Sport Medicine (2006), Gervis, Pickford and Hau (2019) and NICE guidelines (2009). This was exemplified by one club who stated; 'I think that it is crucial and this maybe the only club that has a comprehensive treatment plan that runs alongside the physical rehabilitation'. But this is currently not normal practice within professional English football clubs, despite there being ample resources available (Nesti, 2010). 
Contrary to the current practices identified in this study, only three participants reported wanting psychological support to be solely provided by physiotherapists. This is exemplified by one participant who stated: 'I think an experienced physiotherapist is often in the best position to recognise and assist'. Given the wealth of evidence (Gervis, Pickford and Hau, 2019; Heaney, 2006; Tracey, 2003) one has to question this approach as assumed expertise with limited knowledge could potentially put players' mental health at risk.

Finally, respondents identified the need for more formal education of all support staff so that they are better equipped to properly assess mental health problems: 'Staff dealing with players on a daily basis should have an informed awareness of the signs of psychological illness'.

Education was also cited as a means to reduce the stigma currently attached to psychological support; 'Mental health generally in football is a hugely neglected field- and too often seen as a weakness which can often lead to a player being regarded as unstable and untrustworthy'. Indeed, it was reported that psychological support should be rebranded as 'performance coaching' this suggests little has changed since Pain and Harwood's (2004) findings over 10 years ago. These findings suggest that more education of the role of psychologist in treating injured players is needed to create an environment where this is valued and perceived as the norm.

Despite many important findings, the present study was not without limitations. Although the questionnaire contributed to our knowledge of the psychology services and support mechanisms currently routinely available for long-term injured footballers, certain methodological issues must be addressed. A lack of definitions may have been problematic. As the term "psychological screening” was not defined it is unclear whether all participants used the same definition. Medics may have responded differently if they were all given the same definition. Important details may have also been lost because certain questions were not 
qualified with additional information. For example, although 55\% of participants reported using interviews to screen for psychological issues, it was not known who was carrying out these interviews or what form they took. An interview carried out by a physiotherapist is likely to be very different to one with a psychologist. Furthermore, although the findings indicated what percentage of clubs had part-time sport psychologists working as support staff further details about how often they worked was not obtained. A part-time sport psychologist working twice a week would have a completely different level of input to one who worked twice a year. Consequently, the ability to interpret this data with more detail was lost. Clearly this warrants further investigation to see when and how sport psychologists are treating longterm injured players and what psychological protocols they are using.

\section{Practical Implications}

The evidence appears to show that there is limited engagement with routine psychological support practises for long-term injured players. Long-term injured players were generally only treated by medical staff without psychological expertise. A further implication from our findings is that where there are no psychological staff, psychological screening rarely takes place. The implication therefore is that clubs need to have competent, appropriately trained psychological staff routinely present.

Furthermore, many do not have formal procedures in place to support long-term injured players. A lack of support mechanisms may be explained by a lack of training of medical staff in the role of psychology in sport injury and a culture within football where psychological support is stigmatised and not understood. However, further research is needed to investigate this issue.

In conclusion, the evidence demonstrates that key stakeholders must work together to create protocols to ensure that psychological support is provided for long-term injured 
footballers. These protocols should aim to increase the number of appropriately qualified psychological professionals treating long-term injured players, create written procedures for screening and referral and ensure mental health is included in medical insurance. It is in clubs' interests to invest in psychological support procedures because research has shown that psychological interventions are effective at improving mental health and the quality and rate of rehabilitation (Podlog et al., 2014; Santi \& Pietrantoni, 2013). Clubs would spend less money on medical services if players spent less time in rehabilitation, were re-injured less often, had fewer problems with future performance, were psychologically healthy and spent more time competing and performing on the pitch. However, unless organisational, educational and cultural changes are made to improve psychological support for long-term injured footballers, many will remain unprotected and vulnerable to the numerous negative psychological consequences of injury. Consequently, there should be a greater insistence on the part of organisations who represent players that clubs are mandated to make involvement of a psychological professional a standard part of prevention and rehabilitation for all injured players. Lastly, the duty of care clubs show towards their injured players is deficient, neglectful and possibly negligent. Every effort needs to be made to enforce the recommendation of both NICE Guideline (2009) and The American College of Sport Medicine (2006) in an endeavour to protect these vulnerable players. 


\section{References}

American College of Sports Medicine, (2006). Psychological issues related to injury in athletes and the team physician: A consensus statement. Medicine \& Science in Sport \& Exercise, 38(11), 2030-2034. doi:10.1249/mss.0b013e31802b37a6

Ardern, C. L., Taylor, N. F., Feller, J. A., \& Webster, K. E. (2012). Fear of re-injury in people who have returned to sport following anterior cruciate ligament reconstruction surgery. Journal of Science and Medicine in Sport, 15, 488-495. doi: 10.1016/j.jsams.2012.03.015

Arvinen-Barrow, M., Hemmings, B., Weigand, D., Becker, C., \& Booth, L. (2007). Views of chartered physiotherapists on the psychological content of their practice: A follow-up survey in the UK. Journal of Sport Rehabilitation, 16, 111-121. Retrieved from http://journals.humankinetics.com/journal/jsr

Arvinen-Barrow, M., Massey, W. V., \& Hemmings, B. (2014). Role of sport medicine professionals in addressing psychosocial aspects of sport-injury rehabilitation: Professional athletes' views. Journal of Athletic Training, 49, 764-772. doi: $10.4085 / 1062-6050-49.3 .44$

Brewer, B. W. (2010). The role of psychological factors in sport injury rehabilitation outcomes. International Review of Sport and Exercise Psychology, 3, 40-61. doi: $10.1080 / 17509840903301207$

Carson, F., \& Polman, R. C. (2008). ACL injury rehabilitation: A psychological case study of a professional rugby union player. Journal of Clinical Sport Psychology, 2, 71-90. Retrieved from http://journals.humankinetics.com/jcsp

Clement, D., Arvinen-Barrow, M., \& Fetty, T. (2015). Psychosocial responses during different phases of sport-injury rehabilitation: A qualitative study. Journal of Athletic Training, 50, 95-104. doi: 10.4085/1062-6050-49.3.52 
Gervis, M., Pickford, H. \& Hau, T., (2019). 'Professional Footballers' Association Counsellors' perceptions of the role long-term injury plays in mental health issues presented by current and former players', Journal of Clinical Sport Psychology, https://doi.org/10.1123/jcsp.2018-0049, in press.

Harris, L. L., Demb, A., \& Pastore, D. L. (2005). Perceptions and attitudes of athletic training students toward a course addressing psychological issues in rehabilitation. Journal of Allied Health, 34, 101-109. Retrieved from http://www.asahp.org/publications/journal-of-allied-health/

Hawkins, R. D., \& Fuller, C. W. (1999). A prospective epidemiological study of injuries in four English professional football clubs. British Journal of Sports Medicine, 33, 196203. doi: 10.1136/bjsm.33.3.196

Hawkins, R. D., Hulse, M. A., Wilkinson, C., Hodson, A., \& Gibson, M. (2001). The association football medical research programme: An audit of injuries in professional football. British Journal of Sports Medicine, 35, 43-47. doi: 10.1136/bjsm.35.1.43

Heaney, C. (2006). Physiotherapists’ perceptions of sport psychology intervention in professional soccer. International Journal of Sport and Exercise Psychology, 1, 67-80. Retrieved from http://www.tandfonline.com/toc/rijs20/current

Heijne, A., Axelsson, K., Werner, S., \& Biguet, G. (2008). Rehabilitation and recovery after anterior cruciate ligament reconstruction: patients' experiences. Scandinavian journal of medicine \& science in sports, 18(3), 325-335.

Hsu, C. J., Meierbachtol, A., George S. Z. \& Chmielewski, T. L., (2017) 'Fear of re-injury in athletes: Implications for rehabilitation', Sports Health, vol. 9:2

Johnston, L. H., \& Carroll, D. (1998). The context of emotional responses to athletic injury: A qualitative analysis. Journal of Sport Rehabilitation, 7, 206-220. Retrieved from http://journals.humankinetics.com/jsr 
Lentz, T. A., Zeppieri, G., George, S. Z., Tillman, S. M., Moser, M. W., Farmer, K. W., \& Chmielewski, T. L. (2014). Comparison of physical impairment, functional, and psychosocial measures based on fear of reinjury/lack of confidence and return-tosport status after ACL reconstruction. The American Journal of Sports Medicine, 43, 345-353. doi: 10.1177/0363546514559707

Manuel, J. C., Shilt, J. S., Curl, W. W., Smith, J. A., Durant, R. H., Lester, L., \& Sinal, S. H. (2002). Coping with sports injuries: An examination of the adolescent athlete. Journal of Adolescent Health, 31, 391-393. Retrieved from http://www.jahonline.org/

Nesti, M. (2010). Psychology in football: Working with elite and professional players. Oxon, UK: Routledge.

NICE Guidance: Depression in adults with a chronic physical health problem: recognition and management, (2009, October), retrieved from https://www.nice.org.uk/guidance/cg91

Niven, A. (2007). Rehabilitation adherence in sport injury: Sport physiotherapists' perceptions. Journal of Sport Rehabilitation, 16, 93-110. Retrieved from http://journals.humankinetics.com/journal/jsr

Pain, M. A., \& Harwood, C. G. (2004). Knowledge and perceptions of sport psychology within English soccer. Journal of Sports Sciences, 22, 813-826. doi: $10.1080 / 02640410410001716670$

Podlog, L., \& Eklund, R. C. (2006). A longitudinal investigation of competitive athletes' return to sport following serious injury. Journal of Applied Sport Psychology, 18, 4468. doi: 10.1080/10413200500471319

Podlog, L., \& Eklund, R. C. (2007). The psychosocial aspects of a return to sport following serious injury: A review of the literature from a self-determination perspective. Psychology of Sport and Exercise, 8, 535-566. doi: 10.1016/j.psychsport.2006.07.008 
Podlog, L., Heil, J., \& Schulte, S. (2014). Psychosocial factors in sports injury rehabilitation and return to play. Physical Medicine and Rehabilitation Clinics of North America, 25, 915-930. doi: 10.1016/j.pmr.2014.06.011

Premier League. (2011). Elite Player Performance Plan. Retrieved from

\section{https://www.goalreports.com/EPLPlan.pdf}

Prentice, W.E., Arnheim, D.D. (2011), Arnheim's Principles of Athletic Training: A Competency-Based Approach. 14th ed. New York, NY: McGraw-Hill Higher Education.

Putukian, M. (2016). The psychological response to injury in student athletes: A narrative review with a focus on mental health. British Journal of Sports Medicine, 50, 145148. doi: 10.1136/bjsports-2015-095586

Ruddock-Hudson, M., O’Halloran, P., \& Murphy, G. (2012). Exploring psychological reactions to injury in the Australian Football League (AFL). Journal of Applied Sport Psychology, 24, 375-390. doi: 10.1080/10413200.2011.654172

Santi, G., \& Pietrantoni, L. (2013). Psychology of sport injury rehabilitation: A review of models and interventions. Journal of Human Sport and Exercise, 8, 1029-1044.

Simms, L. J., Zelazny, K., Williams, T. F., \& Bernstein, L. (2019). Does the number of response options matter? Psychometric perspectives using personality questionnaire data. Psychological Assessment. Advance online publication. doi: $10.1037 /$ pas0000648

Smith, A. M., \& Milliner, E. K. (1994). Injured athletes and the risk of suicide. Journal of Athletic Training, 29, 337-341. Retrieved from http://natajournals.org/

Sundgot-Borgen, J. (1994). Risk and trigger factors for the development of eating disorders 
in female elite athletes. Medicine and Science in Sports \& Exercise, 26, 414-419. Retrieved from http://www.acsm.org/public-information/acsm-journals/medicinescience-in-sports-exercise

Tjong, V. K., Murnaghan, M. L., Nyhof-Young, J. M., \& Ogilvie-Harris, D. J. (2014). A qualitative investigation of the decision to return to sport after anterior cruciate ligament reconstruction to play or not to play. The American Journal of Sports Medicine, 42, 336-342. doi: 10.1177/0363546513508762

Tracey, J. (2003). The emotional response to the injury and rehabilitation process. Journal of Applied Sport Psychology, 15, 279-293. doi: 10.1080/714044197

Tripp, D.A., Stanish, W., Ebel-Lam, A., Brewer, B.W., \& Birchard, J. (2007). Fear of reinjury, negative affect, and catastrophizing predicting return to sport in recreational athletes with anterior cruciate ligament injuries at 1 year postsurgery. Rehabilitation Psychology, 52, 74-81. doi: 10.1037/0090-5550.52.1.74 